\title{
¿QUÉ PENSAMIENTO PROFESIONAL Y CURRICULAR TIENEN LOS FUTUROS PROFESORES DE CIENCIAS DE SECUNDARIA?
}

\author{
MARTÍNEZ AZNAR, M.M. ${ }^{1}$, MARTÍN DEL POZO, R. ${ }^{1}$, RODRIGO VEGA, M. ${ }^{1}$, \\ VARELA NIETO, M.P. ${ }^{1}$, FERNÁNDEZ LOZANO, M.P. ${ }^{2}$ y GUERRERO SERÓN, A. ${ }^{3}$ \\ ${ }^{1}$ Departamento de Didáctica de las Ciencias Experimentales \\ ${ }^{2}$ Departamento de Psicología Evolutiva y de la Educación \\ ${ }^{3}$ Departamento de Sociología VI \\ Facultad de Educación. Universidad Complutense de Madrid. Rector Royo Villanova, s/n. 28040 Madrid
}

\begin{abstract}
SUMMARY
This article is a part of a wider research on secondary science teachers' beliefs. Through the use of a questionnaire data were obtained from 211 secondary science teacher candidates. Analyses of data are discussed in terms of their implication in science teacher education.
\end{abstract}

\section{INTRODUCCIÓN}

A partir de mediados de los años setenta, el interés de la investigación sobre el profesor se fue desplazando desde el estudio de su conducta y de la formación basada en competencias técnicas para la enseñanza hacia el estudio de su pensamiento y de la formación basada en un proceso constructivo que incida en cómo piensan $\mathrm{y}$ actúan los profesores (Yinger, 1986). Diez años después, en esta línea de investigación sobre el pensamiento del profesor se observa un progresivo aumento de los estudios sobre lo que los profesores piensan, acerca de la ciencia, su enseñanza y aprendizaje, en general y con relación a contenidos escolares de ciencias en particular, que son de gran interés para orientar nuestro trabajo sobre el pensamiento curricular de los futuros profesores de secundaria (Shulman, 1986; Furió, 1994; Porlán y Rivero, 1998; Wideen et al., 1998).

Concretamente, son numerosos los estudios que señalan la inadecuación del conocimiento de los profesores, tanto en formación inicial como en activo, acerca del conocimiento científico.
Distintos trabajos detectan que los profesores tienden a exaltar la supremacía del conocimiento científico sobre otros conocimientos (Lederman, 1992; Gallagher, 1991; 1993). Otros, como el de Porlán (1989), con muestras de estudiantes de ciencias de magisterio y profesores de ciencias de primaria en activo, revelan una cierta diversidad de concepciones sobre la ciencia. Este autor destaca tres tendencias epistemológicas en relación con ello: racionalismo, relativismo y empirismo. Esta última opción es mayoritaria entre los docentes investigados mientras que las posturas relativistas serían escasas, o sea, apenas se daría importancia a la influencia de los sujetos en la producción del conocimiento científico y al carácter provisional y evolutivo de éste. Otros trabajos, como los de Kouladis y Ogborn $(1989,1995)$, manifiestan la diversidad de posturas dentro del pensamiento sobre la ciencia entre el profesorado; así, los puntos de vista de éste con frecuencia no son totalmente coherentes en todas las dimensiones o aspectos estudiados sobre la ciencia (naturaleza, estatus, producción, cambio, etc.), destacando un punto de vista ecléctico sobre el conoci- 
miento científico. También los resultados de Mellado (1996) apuntan a la inexistencia entre los profesores de una concepción definida y coherente sobre el conocimiento científico. En todo caso, como señala este autor, la posible influencia de estas concepciones sobre la actuación docente está mediatizada por otros muchos factores complejos, siendo lo más destacable la escasa formación epistemológica del profesorado.

En resumen, a pesar de la diversidad de concepciones, frecuentemente se plantean visiones del conocimiento científico como superior, objetivo, neutral y descontextualizado. Esto para algunos autores (Porlán y Martín del Pozo, 1996; Porlán et al., 1998) sería un serio obstáculo para la mejora del conocimiento profesional.

Por otra parte, distintos autores (Hollon y Anderson, 1987; Porlán, 1989; Prawat, 1992; Hewson y Hollon, 1994; López, 1995) ponen de manifiesto la importancia que tienen las concepciones de los profesores sobre el aprendizaje. En dicho sentido se situarían creencias como:

- Los alumnos aprenden si están atentos a la explicación del profesor y estudian. Si no lo hacen, es porque estudian poco o tienen problemas familiares.

- Ese aprendizaje se manifiesta como lo que son capaces de explicar en los exámenes.

- Si se tienen en cuenta las ideas de los alumnos es para cambiarlas por la «verdad científica».

De forma similar a lo que sucede con las concepciones sobre la ciencia, algunos estudios muestran que los profesores no poseen siempre concepciones uniformes y coherentes sobre el aprendizaje (Desaultes, 1993; Hewson et al., 1995). No obstante, las posiciones de recepción por «transmisión verbal del conocimiento» o de adquisición «por simple actividad de los alumnos» son bastante frecuentes. Por contra, el desarrollo de visiones constructivistas, que son las consideradas de mayor interés educativo, implicaría una evolución paulatina de los docentes y estarían representadas minoritariamente dentro de este colectivo (Porlán et al., 1997 y 1998). No obstante, incluso cuando se manifiestan este tipo de concepciones, tampoco son tan uniformes; así en el estudio de cuatro casos realizado por Mellado (1996) se detectan, en todos ellos, diferentes grados de una visión constructivista del aprendizaje.

En lo referente a las concepciones acerca de la enseñan$z a$, parece existir una correspondencia entre éstas y las relacionadas con el aprendizaje. Así, Kennedy (1991, p. 7), revisando distintos trabajos sobre profesores pone de manifiesto la supremacía de la concepción de que el aprendizaje supone absorción y la enseñanza contar a los estudiantes lo que el profesor conoce, así como evaluar el recuerdo de los estudiantes de este conocimiento. Porlán (1989) estudiando a futuros profesores y a profesores en activo detectó una variedad de modelos didácticos:
- Modelo tecnológico, en el que los objetivos eran concebidos como el elemento estructurador tanto de la práctica como de la evaluación del aprendizaje de los alumnos. Así se concibe la enseñanza como una actividad técnica que debe asumir métodos didácticos científicos. Este modelo predominaba entre los futuros profesores de EGB estudiados. Asimismo, este hecho se evidencia en el estudio de Pérez Gómez y Gimeno (1992) en el que estudiaban a futuros profesores de secundaria que realizaban el CAP (Curso de Aptitud Pedagógica).

- Modelo tradicional, caracterizado por la transmisión verbal de los contenidos disciplinares. Éste parece ser el modelo predominante entre el profesorado en activo.

- Modelo alternativo, en el que se resaltaría la participación de los alumnos y la investigación del profesor, siendo este planteamiento minoritario.

Modelos similares aparecen en otros trabajos realizados por autores como Marrero (1993) y Gallagher (1991). Podemos, pues, decir que las posturas tradicionales son predominantes; no obstante, se detectan planteamientos tecnológicos, sobre todo, entre aspirantes a profesores formados en este modelo didáctico. Otros planteamientos más activistas y con posturas más próximas al constructivismo serían minoritarios a pesar de ser consideradas propias de un desarrollo profesional deseable (Porlán et al., 1997, 1998).

¿Qué contenidos, metodología o evaluación son los más adecuados cuando se enseña ciencias? El estudio de las concepciones curriculares sobre tópicos concretos de ciencias es una línea de trabajo de creciente interés; en ese sentido se sitúa el trabajo efectuado por Martín del Pozo (1994), en el cual, analizando las unidades didácticas elaboradas por 24 estudiantes de ciencias de magisterio sacaba, entre otras, las siguientes conclusiones:

- Los contenidos eran considerados como un conjunto acumulativo y fragmentario de conceptos, leyes y teorías que se organizan en forma de listas desconexionadas. En su selección, el libro de texto es la clave. A veces, y de una manera minoritaria entre los investigados, aparecen tendencias a recoger contenidos con aplicación en la vida diaria.

- Respecto a la metodología, existe una tendencia generalizada a ligar actividad -por ejemplo, de observacióncon aprendizaje. Asimismo, correlacionan la explicación del profesor con el aprendizaje de los alumnos. Generalmente se tiende a concebir la metodología de trabajo como una secuencia única y cerrada de actividades. En un plano más positivo, en algunos casos, se pone atención a los intereses e ideas de los alumnos.

- La evaluación es considerada como comprobación de aprendizajes conceptuales utilizando básicamente pruebas escritas. A veces aparecen otras tendencias como: una orientación tecnológica (con prueba inicial y final); o una orientación espontaneísta (no incluyendo exámenes de evaluación y sólo evaluando por actitudes); o un 
planteamiento complejo, promoviendo una evaluación continua de estudiantes y profesores pero sin clarificarla.

Pérez Gómez y Gimeno (1992) estudiando a alumnos que realizaban el Curso de Aptitud Pedagógica (CAP) pusieron de manifiesto creencias semejantes a las que acabamos de ver en cuanto a contenidos y evaluación. Por otra parte, en estudios con profesores en activo (Cronin Jones, 1991; Pérez Gómez y Gimeno, 1992) se evidencian, por un lado, concepciones abiertas en cuanto a metodología y contenidos, pero con tendencias tradicionales en cuanto a evaluación. En esta línea de manifiestas incoherencias entre selección de contenidos y propuestas metodológicas a aplicar se situaría lo observado por Hollon, Roth y Anderson (1991). En otros casos incluso se manifiesta una cierta diversidad metodológica entre profesores que dicen situarse en una misma línea de trabajo basada en la investigación del alumno (Orlandi, 1991). También en el estudio de Mellado (1996) se aprecia esta diversidad, más aún cuando se trata de buscar la correspondencia entre las declaraciones y las actuaciones en el aula, pues aparecen todas las situaciones posibles: coherencia total, parcial y prácticamente inexistente.

Por último, cabría preguntarse de una manera sintética si se pueden relacionar la manera de enfocar la enseñanza de los profesores de ciencias con sus concepciones epistemológicas. En este sentido, se situarían los primeros estudios como los de Pope y Scott (1988) en los que se intentan relacionar las concepciones positivistas y empiro-inductivistas de la ciencia con versiones absolutistas de la verdad y del conocimiento. Todo ello llevaría a propuestas curriculares en las que las ideas de los alumnos y su participación en la tarea docente no son tenidas en cuenta. En la línea anterior, se situarían estudios como los de Pomeroy (1993), Smith y Neale (1991), Gustafson y Rowell (1995) y Hashweh (1996). También aparece con fuerza la idea de que las maneras de interpretar la ciencia podrían constituir un obstáculo relevante para la progresión del conocimiento profesional deseable (Porlán, 1989). No obstante a lo dicho anteriormente, debemos recordar que hay autores como Prawat (1992) que critican estas correlaciones planteadas de una manera tan directa. De hecho, por ejemplo, en los resultados del estudio de Mellado (1996) no se detecta una correspondencia entre las concepciones sobre la ciencia y las de su enseñanza y aprendizaje.

Queremos indicar que, por otra parte, nuestro interés por este tema de investigación no está separado de nuestra actividad docente como formadores de profesores. Así, la consideración de las concepciones de los estudiantesprofesores a lo largo del proceso de formación inicial constituye un principio formativo de gran potencialidad que supone una clara plasmación del principio de coherencia entre el modelo de formación y el modelo didáctico en él contenido. Ambos responden a un planteamiento constructivista en el aprendizaje de conocimientos, sean éstos de índole escolar o profesional. Dicho en otros términos, en la formación inicial no se puede plantear como un conocimiento profesional trabajar didácticamente con las concepciones de los alumnos sin trabajar con las concepciones de los estudiantes-profesores en el proceso de formación. Si logramos hacer conscientes a los futuros profesores de esta isomorfía, sin duda aquí el medio (trabajar con las concepciones de los estudiantesprofesores) está siendo también el mensaje (trabajar con las concepciones de los alumnos). Estas consideraciones se establecen tanto con las concepciones sobre el contenido conceptual (sobre cualquier concepto de ciencias incluido en el currículo escolar de secundaria) como a las de contenido didáctico (sobre qué y cómo enseñar y evaluar un contenido concreto de ciencias). Hemos de tener en cuenta que los futuros profesores de secundaria ya tienen un determinado conocimiento de alguna de las ciencias de la naturaleza -adquirido durante su licenciatura- que no es específico para enseñar sobre dichas disciplinas; es decir, no es un conocimiento profesionalizado cuya práctica social de referencia sea la enseñanza, sino la investigación científica (Martín del Pozo, 1994). Por otra parte, es evidente que no tienen unos conocimientos prácticos sobre la enseñanza que estén generados por su propia experiencia profesional, como ocurre con los profesores con relación a la formación permanente. Ahora bien, manifiestan un cierto tipo de conocimiento para actuar, para lo que habitualmente se entiende como «dar clase», que podría estar generado por el recuerdo de la actuación de sus profesores desde su experiencia como alumnos. Según Furió y otros (1992), este tipo de conocimientos constituye un pensamiento docente de sentido común adquirido por formación ambiental durante la escolaridad, que tiene una gran influencia en la actuación futura y que no suele alterarse con la formación inicial (Knowles, 1992; Mellado, 1998).

Además, con todos estos datos de la investigación sobre las concepciones de los profesores, se puede establecer una gradación en la construcción del conocimiento profesional desde perspectivas más simplificadoras, que podrían concretarse en los modelos didácticos tradicionales, hasta otras más constructivistas; en cualquier caso, se dan distintos modelos intermedios. Por ejemplo, podemos recordar el análisis de modelos de profesor de ciencias propuesto por Fernández y Elortegui (1996), en el cual, comparando los distintos planteamientos curriculares que aparecen entre el profesorado, surgirían los siguientes modelos: transmisor o tradicional, tecnológico, artesano, descubridor y constructor. Asimismo, sería interesante recordar las hipótesis de progresión del conocimiento profesional planteadas últimamente por autores como Porlán y otros (1998). En general, todas estas hipótesis podrían servir para orientar los procesos de formación del profesorado.

Para concluir y desde otro punto de vista, los estudios sociológicos sobre el profesorado parecen poner de manifiesto relaciones entre los contextos socioprofesionales, y el pensamiento y la práctica docentes. En la revisión de los diferentes enfoques para estudiar al profesorado en España y centrándose en el enfoque sociológico, Barquín (1995, p. 19) concluye que el retrato del profesor que se deriva de la perspectiva sociológica es claramente restrictivo cuando no catastrofista, amén de detectarse una tendencia a la baja en 
la calidad académica y procedencia social de los futuros docentes. Esto estaría en consonancia con datos como que la extracción social de los enseñantes es fundamentalmente de clases medias-bajas; es decir, la profesión de enseñante parece que no atrae a las capas de población más formadas y económicamente más solventes. Además, se constataría un fuerte incremento en la presencia de mujeres entre los enseñantes; esto último, algunos lo asocian con la pérdida de prestigio, menos capacidad reivindicativa y una adecuación sexista del trabajo femenino al ámbito educativo (García Yagüe, 1988). Parece, entonces, necesario que en la formación del profesorado también se cuente con datos acerca de los estudiantes-profesores como grupo social y sobre cómo entienden su profesión para poder intervenir de manera eficaz de cara a potenciar profesionales críticos.

En resumen, el estudio de las concepciones científicas, didácticas y profesionales de los futuros profesores, además de ser una prometedora línea de investigación, es una necesidad para la prácticëTen formación del profesorado, puesto que dichas concepciones, entendidas como un conocimiento alternativo a dichos conocimientos, constituyen la información a movilizar en el proceso de formación inicial. En esta línea aportamos los resultados más relevantes del estudio realizado con estudiantes del Curso de Aptitud Pedagógica (CAP) de la Universidad Complutense de Madrid para indagar sus concepciones curriculares en el área de ciencias de la naturaleza. También nos proponemos caracterizar social y profesionalmente a dicho colectivo y finalmente, planteamos algunas sugerencias para la formación inicial del profesorado de secundaria de cara a la nueva modalidad institucional, el Curso de Cualificación Pedagógica, ya implantada en algunas universidades.

\section{PLANIFICACIÓN Y DESARROLLO DE LA INVESTIGACION}

De acuerdo con el planteamiento de la investigación y dentro del marco teórico propuesto, el objetivo general de este estudio ha sido describir y analizar el pensamiento del futuro profesor de ciencias de educación secundaria en dos ámbitos: uno en relación con aspectos profesionales y otro sobre aspectos curriculares. Para ello hemos trabajado con una muestra de 211 sujetos, seleccionada aleatoriamente entre los futuros profesores de ciencias que habían realizado el Curso de Aptitud Pedagógica (CAP) en la UCM durante el curso 1996-97. Esta población estaba constituida por 692 alumnos de la modalidad de ciencias naturales y 627 de la modalidad de física y química (1.319 individuos).

Estos cursos conducentes a la obtención del Certificado de Aptitud Pedagógica tienen las siguientes características: "metodología autoformativa, asesoramiento mediante tutorías y evaluación personalizada. La formación se complementa con la realización de prácticas en centros educativos para quienes carezcan de esta experiencia y la realización de una memoria de prácticas que integre los aprendizajes logrados en la formación teórica y en la experiencia práctica... En resumen, el alumno, una vez que tiene en su poder los temas, dedica el tiempo disponible a su aprendizaje; es ayudado, en caso de dudas, por tutores, a los que puede consultar telefónicamente o de forma presencial. Posteriormente, el alumno se examina del bloque correspondiente de aprendizaje, en la fecha indicada y recibe a continuación los resultados». (Guía de Estudio para el CAP del ICE de la UCM, 1997, pp. 1-2).

El curso se divide en módulos: seis comunes (Educación y sistema educativo; Psicología del aprendizaje y del desarrollo; Acción tutorial; Innovación e investigación educativa en el aula; Tecnología educativa y Programación y Evaluación Curricular) y uno específico (Didáctica de Física y Química o Didáctica de Ciencias Naturales). Además se imparten seminarios presenciales vinculados a la Didáctica Específica y Prácticas, de 30 horas, a realizar en situaciones reales en centros educativos. Los requisitos exigidos para la obtención del certificado implican superar un examen sobre los módulos ya indicados, asistir a los seminarios y presentar la memoria de las prácticas.

\section{Metodología de la investigación}

Este trabajo se ha desarrollado mediante un estudio descriptivo, ex post facto, que nos ofrece una caracterización de los alumnos del CAP (Área de Ciencias). Para la investigación más amplia, de la que ésta forma parte (Martínez et al.,1997), se emplearon metodologías cuantitativas y cualitativas de acuerdo con las necesidades específicas del diseño. En particular, en este trabajo se ha utilizado una metodología cuantitativa por ser la que nos permite responder a los objetivos planteados que son predominantemente descriptivos (Esteve, 1997). Más concretamente, se requería un instrumento que permitiese precisar las ideas que el estudiante del CAP manifiesta sobre su posible labor docente, es decir, referentes a sus intenciones como futuro profesional (lo que piensa que se debe hacer). Teniendo en cuenta distintos trabajos empíricos en los que se han desarrollado instrumentos para estudiar el pensamiento del profesor (Barquín, 1991; Pérez Gómez y Gimeno, 1992; Marcelo, 1995; Porlán et al., 1998), se elaboró el cuestionario que aparece en el anexo. Su versión definitiva se redactó a finales del curso 1995-96, previa consulta a expertos y la realización de un ensayo piloto que permitió poner a prueba el instrumento, reformular de nuevo algunos ítems y eliminar aquéllos poco discriminativos (Azorín y Sánchez Crespo, 1986). No obstante, somos conscientes de las limitaciones de un cuestionario para detectar las concepciones de los profesores (Mellado, 1996; Gunstone et al., 1993; Lederman y O'Mally, 1990), tanto en lo referente a su potencialidad a la hora de reflejar el pensamiento de los encuestados, la interpretación que los sujetos hacen de los enunciados de los ítems como en la propia estructura que se adopta en la elaboración de dimensiones, categorías y número de proposiciones planteadas. La construcción de los ítems se ajusta en su mayoría a la modalidad escalar de Likert, y en su elabo- 
ración hemos tenido en cuenta las normas propuestas en 1957 por Edwards (Triandis, 1971). En el cuestionario se han considerado dos grandes bloques:

a) uno centrado en los aspectos profesionales estudiados a través de 6 preguntas de libre elección y 14 ítems;

b) otro integrado por 51 declaraciones relativas a aspectos curriculares, cuyas posibles respuestas son: «totalmente de acuerdo», «de acuerdo», «indeciso», «en desacuerdo»y «totalmente en desacuerdo».

En el bloque centrado en los aspectos profesionales pueden distinguirse:

- Para la identificación de los sujetos, las señas de identidad básica pasan por la ubicación de los encuestados en relación con la edad, el sexo y el origen social (preguntas $a, b$ y $c$ del cuestionario). Los grupos de edad se han construido según intervalos de cinco años, que parece el más conveniente para estudiar la significación de esta variable. La variable sexo se ha incluido desde la perspectiva de los estudios de género, en la medida en que la construcción social de las diferencias de sexo podrían explicar actitudes y comportamientos distintos; el hecho cobra especial relevancia dentro de una profesión que se considera en proceso creciente de feminización. El origen social se plantea tanto desde la perspectiva demográfica en relación con la posición social de los padres, medida por la profesión de ambos cónyuges como desde sus posibles conexiones con las motivaciones de elección profesional y posteriores actitudes y prácticas docentes. Se ha incluido también dentro de los datos de identificación una variable básica de tipo profesional como es la formación inicial según el nivel de estudios y la especialidad cursada por los encuestados (pregunta $d$ ).
- Desde el punto de vista de las percepciones profesionales, en las preguntas $e$ y $f$ se indaga acerca de la mayor o menor amplitud de los modelos profesionales docentes, recogiendo indicadores relativos al grado de influencia que ejercen sobre este trabajo diversos agentes sociales e instituciones educativas y que constituyen uno de los tópicos más reiterados entre los profesores al hablar de educación. Por otra parte, se han elaborado una serie de ítems para explorar un conjunto de ideas o actitudes relativas a: a) la formación inicial recibida tanto en el aspecto científico como psicopedagógico (ítems 48 y 49); b) la incidencia de una serie de factores sociales y personales tanto del profesor como del alumno sobre el rendimiento de estos últimos (ítems 50, 51, $52,55,56,57,60$ y 62); c) características de las materias de ciencias (ítems 53, 61, 63 y 64). En el cuadro I se recogen los aspectos de este bloque del cuestionario.

En el bloque relativo a los aspectos curriculares y para determinar sus componentes, hemos adoptado los interrogantes genéricos: ¿Qué enseñar? ¿Qué, cómo y cuándo evaluar? ¿Cómo enseñar? Dichos interrogantes se hacen corresponder con: dimensión de contenidos, dimensión de evaluación y dimensión de metodología respectivamente. Dada la amplitud de aspectos que se pueden incluir en cada dimensión, nos hemos limitado a los que habitualmente son tratados por otros autores (Rodrigo, 1994; Pérez Gómez y Gimeno, 1992; Barquín, 1991; Carrascosa et al., 1991; Gil, 1991; Marcelo, 1991; Porlán, 1989; Brincones et al., 1986...) y a nuestras propias necesidades e intenciones investigadoras, basadas en la reflexión sobre lo que consideramos relevante para identificar la práctica y el pensamiento docentes (Cuadro II).

En las proposiciones de este bloque se pretende establecer dos tendencias: una concepción constructivista del

Cuadro I

Distribución de las proposiciones del cuestionario para la dimensión profesional.

\begin{tabular}{|c|c|c|}
\hline Dimensión profesional & Aspectos estudiados & Proposiciones del cuestionario \\
\hline IDENTIFICACIÓN & $\begin{array}{l}\text { - Edad, sexo, origen social } \\
\text { - Formación previa }\end{array}$ & $\begin{array}{l}a, b, c \\
d\end{array}$ \\
\hline PERCEPCIONES PROFESIONALES & $\begin{array}{l}\text {-Modelos profesionales } \\
\text {-Percepción de la formación inicial } \\
\text {-Percepción de la incidencia } \\
\text { de factores sociales y personales } \\
\text { sobre el rendimiento escolar } \\
\text { - Percepción de las materias de ciencias }\end{array}$ & $\begin{array}{l}\text { e, f } \\
48,49 \\
50,51,52,55 \\
56,57,60,62 \\
53,61,63,64\end{array}$ \\
\hline
\end{tabular}


Cuadro II

Distribución de las proposiciones del cuestionario para las dimensiones curriculares.

\begin{tabular}{|l|l|l|}
\hline \multirow{2}{*}{ Dimensiones curriculares } & Aspectos estudiados & Proposiciones del cuestionario \\
\hline \multirow{2}{*}{ CONTENIDOS } & - Relación con otros conocimientos & $36,37,38,39,40,41$ \\
& - Fuentes y organización & $42,43,44,45,46,47$ \\
\hline \multirow{2}{*}{ EVALUACIÓN } & - Instrumentos & $1,2,3,4,5$ \\
& - Diseño y organización & $6,7,9,10,11$ \\
& - Finalidad & 8,12 \\
\hline \multirow{2}{*}{ METODOLOGÍA } & - Planificación & $13,14,15$ \\
& - Desarrollo de la enseñanza & $16,17,18,19,20$ \\
& - Adaptación al alumno & $21,22,23,58,59,65$ \\
& - Motivación y participación & $24,25,26,27,28,29,30,31,54$ \\
& - Recursos & $32,33,34,35$ \\
\hline
\end{tabular}

proceso de enseñanza-aprendizaje frente a un enfoque más «reproductivo» o tradicional (Coll, 1994; Driver et al., 1989; Porlán, 1993 y MEC, 1992). Estas tendencias se han determinado considerando que las concepciones o creencias que tienen los profesores presentan una cierta organización y dinámica, y constituyen el punto de partida para la construcción del «conocimiento profesional» (Porlán et al., 1998). Así, la gradación en la construcción de este conocimiento profesional va desde perspectivas más simplificadoras, que podrían concretarse en los modelos didácticos tradicionales, hasta otras más constructivistas, sin olvidar otros posibles modelos intermedios (Fernández y Elortegui, 1996).

El paso siguiente en la elaboración de este bloque fue relacionar los aspectos estudiados con las dos tendencias comentadas (constructivista y tradicional), siendo los extremos de la escala los que permiten una adscripción de los sujetos a una u otra tendencia (Anexo). A modo de ejemplo, dentro de la dimensión Contenidos y para el estudio de la Relación con otros conocimientos, cuando se pretende explorar el papel de las concepciones alternativas en relación con el conocimiento científico, se presentan las siguientes proposiciones: $a$ ) «Las ideas de los alumnos sobre los conceptos de ciencias son errores que el profesor debe eliminar.» (tendencia tradicional, ítem 40); b) «Las ideas de los alumnos sobre los conceptos de ciencias son un conocimiento alternativo con el que hay que trabajar en clase.» (tendencia constructivista, ítem 41).

Para el análisis de los datos obtenidos de las cuestiones con escala tipo Lickert, se han calculado los porcentajes de contestaciones situadas en los extremos de las escalas, es decir, la suma de las respuestas $1+2$ y $4+5$ (totalmente de acuerdo + acuerdo y desacuerdo + totalmente en desacuerdo), seleccionándose aquellas opcio- nes que contestan como mínimo el $70 \%$ de los encuestados y cuya media (calculada de 1 a 5 , totalmente de acuerdo [...] total desacuerdo) esté suficientemente polarizada hacia uno de los extremos de la misma (varianza igual o menor a 0,98). En las preguntas diseñadas en concepto de libre elección se ha realizado un recuento para cada enunciado o contestación dada, con un cálculo del porcentaje relativo de cada respuesta frente al total.

Por otra parte, hay que señalar que el tiempo empleado en la cumplimentación del cuestionario fue libre y, en cualquier caso nunca superior a una hora; en la aplicación siempre estuvo presente algún miembro del equipo investigador como testigo de la seriedad con que se respondió.

\section{RESULTADOS}

De acuerdo con la estructura del cuestionario utilizado en la investigación, vamos a presentar los resultados según las dimensiones definidas para su diseño. En primer lugar, va a aparecer la información correspondiente a las características personales y profesionales de la muestra estudiada mostrándose a continuación todo lo relativo a sus opiniones en el campo de las dimensiones curriculares.

\section{Identificación de los sujetos}

Los datos extraídos de los cuestionarios en relación con la composición por edad y sexo muestran, como era de esperar, la pertenencia de los profesores en formación inicial a una edad joven, deducible de la tendencia 
Gráfico 1

Perfil del alumnado del CAP obtenido a partir del cuestionario «Pensamiento-Contenidos».

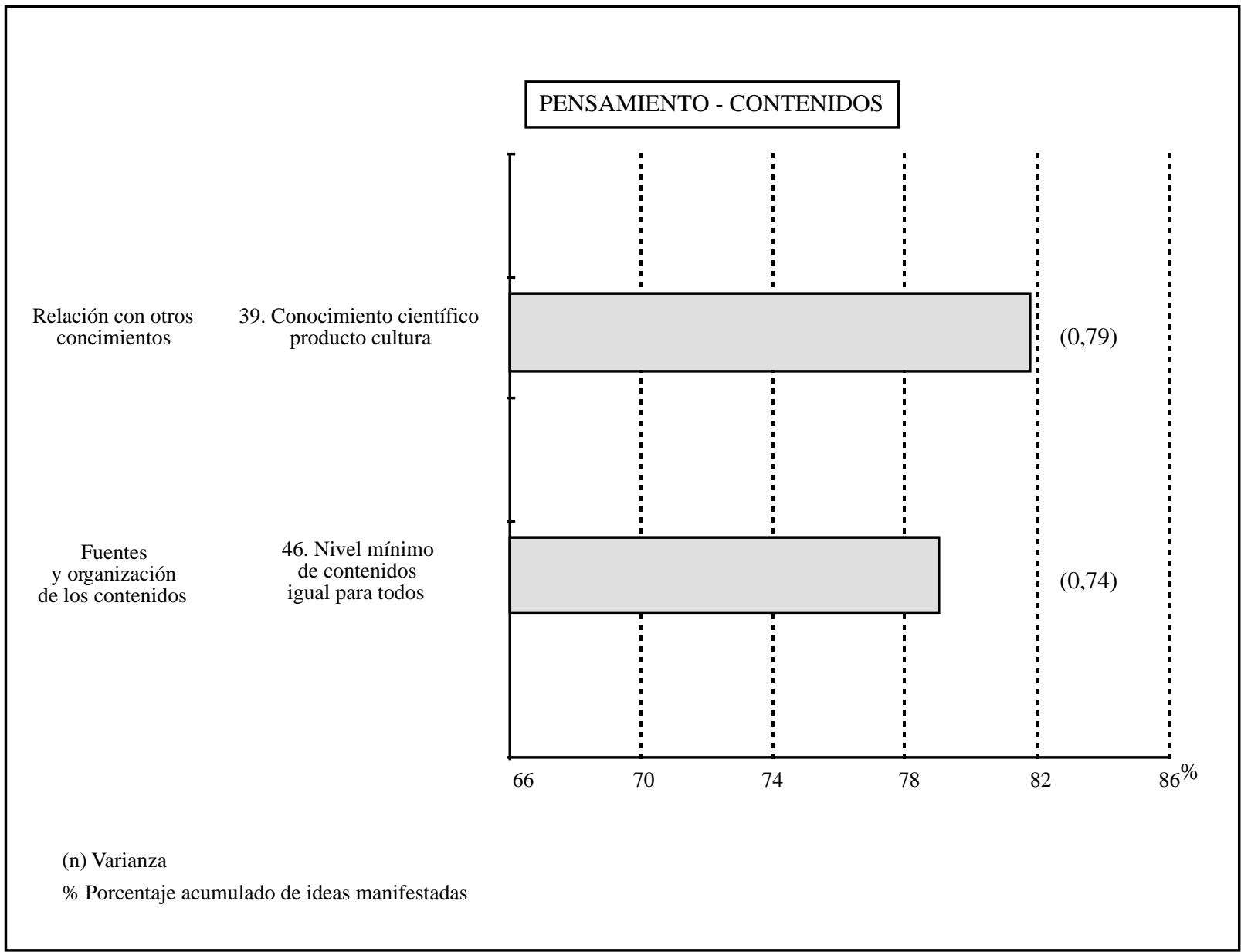

mayoritaria a obtener la capacitación pedagógica inmediatamente después de terminar los estudios de licenciatura. Así, prácticamente el 50\% de los entrevistados tiene menos de 25 años y más de la tercera parte, entre 26 y 30 años. Llama la atención, no obstante, que el 15\% restante se ubique todavía, aunque en proporción progresivamente menor, entre los 31 y los 55 años de edad: no cabe duda de que se trata de vocaciones tardías, en la mayoría de los casos, toda vez que la pertenencia a la docencia concede esa capacitación, no sólo de facto, sino también de iure. Con relación al sexo, se aprecia una presencia femenina muy superior a la masculina: exactamente el doble de mujeres que de hombres. Tales datos están en consonancia con la feminización cuantitativa de la enseñanza universitaria, en general, y la docencia secundaria, en particular (Guerrero, 1997).

Los datos obtenidos de los cuestionarios en lo que se refiere a los orígenes sociales medidos a partir de la ocupación de los padres (pregunta $c$ ) permiten establecer que la procedencia de quienes aspiran a ser profeso- res de ciencias en secundaria viene a ser, en un porcentaje del orden del $84 \%$, la que se conoce -en sentido amplio-como clase media, en especial la llamada nueva clase media, formada por personas que obtienen sus rentas de profesiones que requieren un mediano o alto nivel de cualificación educativa: profesores, funcionarios y otros profesionales.

Por último, cabe indicar que en la población estudiada se da una importante endogamia, encontrándose un porcentaje significativo de hijos e hijas de profesores $(6,8 \%$ teniendo presente que el peso específico de los profesores en el total de la población es del $0,12 \%$ ), que prueba que la reproducción interna en la profesión docente es bastante notable, aunque se dé en menor proporción que en otras profesiones (Bernstein, 1988).

Respecto a la formación previa de los aspirantes a la capacitación pedagógica, pueden destacarse tres hechos significativos. Por orden de importancia, hay que señalar la existencia de una titulación claramente mayoritaria 


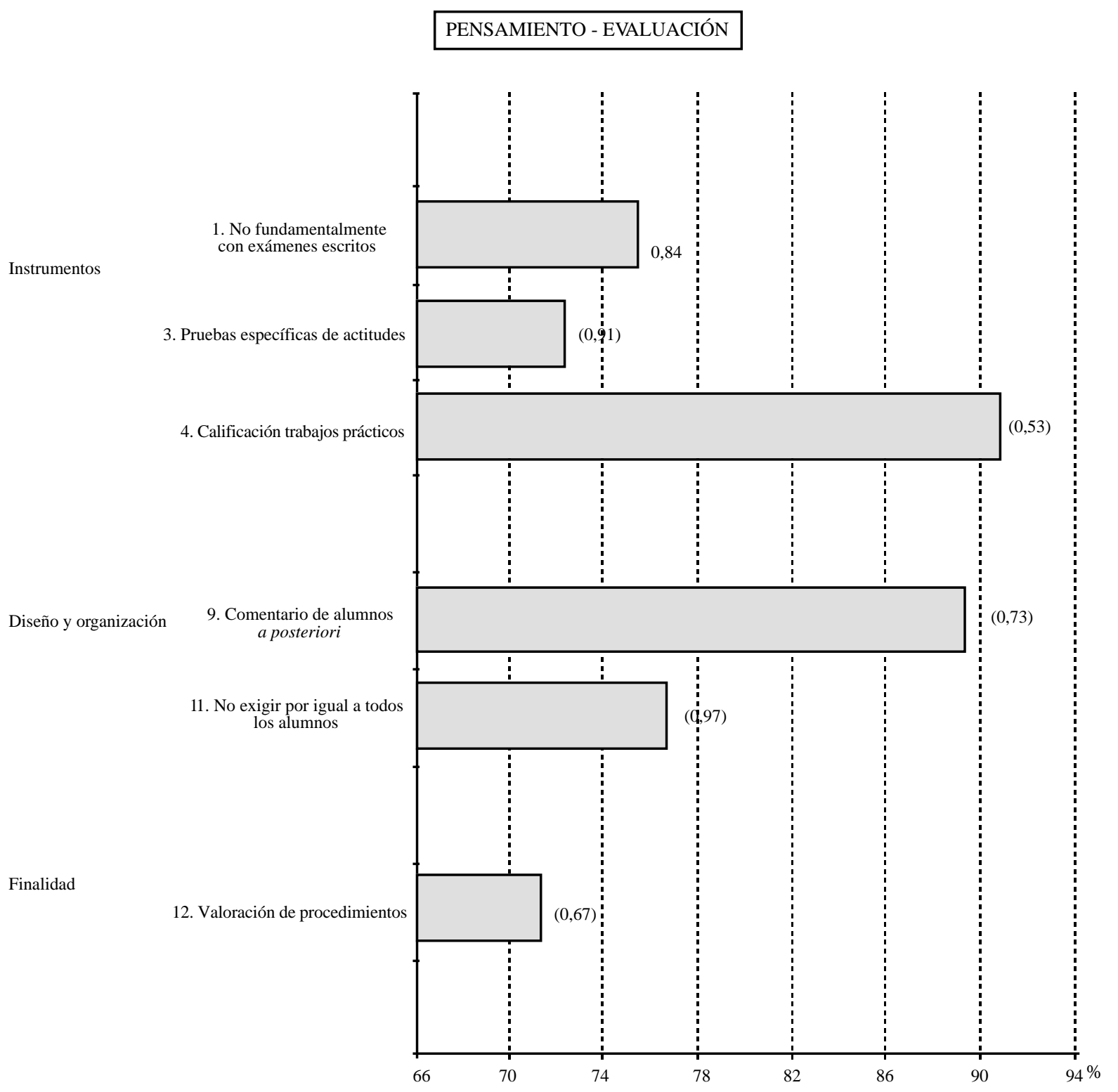

(n) Varianza

$\%$ Porcentaje acumulado de ideas manifestadas

en cada una de las dos especialidades del CAP encuestadas: química y biología. Mientras hay más de dos licenciados en química por cada uno en física que aspira a obtener el CAP, en el caso de ciencias naturales, los biólogos son más del triple que los geólogos. ¿Polivalencia química o colonialismo ecológico-cultural? Quizás una simple cuestión de inadecuación entre formación inicial y mercado laboral.

En cuanto a las licenciaturas específicas o idóneas, éstas no son las únicas que conducen a la docencia en secundaria: además de los titulados esperados, hay también 
farmacéuticos, ingenieros, arquitectos e, incluso, médicos (¡quien lo diría!) entre quienes pretenden acceder a enseñar ciencias en la enseñanza secundaria. Por último, diez de cada cien de los aspirantes a profesor tienen el grado de doctor. La licenciatura, tal cual, no parece ser la única formación aportada por quienes aspiran a ser profesores de ciencias de secundaria. Esto viene a insistir en el carácter credencialista que cada vez más adopta nuestro sistema de enseñanza y la prolongación de la escolaridad como elemento de distinción y competencia en el mercado de las recompensas simbólicas.

\section{Percepciones profesionales}

El estudio de Broadfoot y Osborn (1988), un clásico en este campo, compara los modelos profesionales imperantes entre los profesores de enseñanza pública de Gran Bretaña y los de Francia, y distingue entre la profesionalidad extendida, propia de los primeros, y la profesionalidad restringida, característica de los segundos, que difieren en el nivel de responsabilidad asumida en el ejercicio de su docencia. Con este marco de referencia, de los resultados obtenidos en el trabajo de campo (cuestión $e$ ), se puede decir que entre los aspirantes a profesores investigados se da un modelo de profesional docente muy extenso, es decir, se sienten responsables ante la comunidad educativa en su conjunto y no solamente ante su tarea concreta dentro del aula. Es una conclusión a la que se llega al ver la saturación de todas las categorías exploradas, salvo la inspección, cuando se ha preguntado el grado de influencia y el sentido de la misma que ejercen sobre el trabajo docente diversos agentes e instituciones educativas (influyen positivamente: los alumnos, $92 \%$; el departamento didáctico y la responsabilidad profesional del profesor, 93\%; el claustro y los libros de texto, 84\%; el consejo escolar y los padres, más del 72\%; y los programas oficiales, el $68 \%$ ). Al comparar estos resultados con los de los profesores en ejercicio que también hemos estudiado (Martínez et al., 1997), se puede deducir que su alejamiento de la práctica docente, su inexperiencia podríamos decir, les hace ser más idealistas y carentes de referencias concretas que los profesores en ejercicio. En todo caso, datos de estas características son indicadores importantes a la hora de evaluar y comparar la evolución profesional durante la carrera docente.

Por otra parte, una mirada a las relaciones que establecen entre aprendizaje y sociedad, derivada de las respuestas a la pregunta $f$ del cuestionario, permite seguir considerando de nuevo al profesorado en formación como un grupo lleno de optimismo, dada su general confianza en las instituciones educativas para elevar el nivel de conocimientos del alumnado de secundaria. Sólo dos agentes externos, la socorrida televisión $(62,5 \%)$ y las escasas perspectivas de empleo $(81,7 \%)$ constituyen, en su opinión, las únicas causas del descenso de nivel de conocimientos de ese alumnado.

De acuerdo con los resultados obtenidos en el cuestionario, la muestra de profesores estudiada tiene elaborada una «mentalidad profesional» que podemos describir a partir de las cuatro variables que han resultado estadísticamente significativas. Con relación a la formación inicial, un $85 \%$ de los alumnos del CAP percibe de manera muy acusada la necesidad de una formación psicopedagógica adecuada a la tarea que van a tener que desarrollar (ítem 49); sin embargo, llama la atención que no se haya alcanzado uniformidad cuando se les ha preguntado sobre si su formación científica es suficiente para poder desempeñar el trabajo en la enseñanza secundaria (ítem 48). En lo relativo a la incidencia de factores sociales y personales sobre el rendimiento escolar de los alumnos, ítem 52, manifiestan su desacuerdo sobre que los varones posean mejores aptitudes que las mujeres en el área científica (78\%) y, alrededor del $75 \%$ de los sujetos encuestados expresan su disconformidad en cuanto a la infravaloración de la personalidad y actitudes del profesor a la hora de realizar una tarea eficaz dentro del aula (ítem 60). Por último, al mencionarse las características de las materias científicas (ítem 63), un porcentaje muy elevado $(94 \%)$ percibe de forma muy marcada que la enseñanza de las ciencias tiene, como una de las finalidades importantes, la de potenciar el pensamiento crítico de los alumnos.

A modo de síntesis, podemos concluir, en lo relativo a aspectos de tipo personal y social, que la mitad de los encuestados tiene menos de 25 años y que las mujeres son el doble que los hombres en consonancia con la feminización creciente de la población universitaria española. Sus orígenes sociales están claramente enmarcados en la denominada clase media dándose una importante endogamia y siendo mayoritariamente licenciados en químicas y biológicas. Por último, su modelo profesional está enmarcado en el llamado «de profesionalidad extensa», es decir, se sienten responsables ante toda la comunidad educativa, confiando en las instituciones a la hora de elevar el nivel académico de los alumnos y reconociendo además la necesidad de una formación psicopedagógoca adecuada.

A continuación, se presentan los aspectos del pensamiento curricular del colectivo de profesores en formación inicial encuestados en el cual ha aparecido una uniformidad significativa (en el $55 \%$ de las cuestiones) de acuerdo con los criterios indicados cuando describimos las técnicas de análisis utilizadas.

\section{Dimensión «contenidos»}

En este campo, los estudiantes del CAP presentan una gran homogeneidad cuando se manifiestan sobre dos proposiciones (Gráfico 1). En lo relativo a la relación del conocimiento escolar con otros contenidos consideramos el conocimiento científico como un producto de la actividad humana y del contexto cultural, alejándose por tanto de una idea rígida sobre el concepto de ciencia $(81 \%)$. En cuanto a la organización y selección de los contenidos, al contestar el ítem 46, el $79 \%$ manifiesta la necesidad de seleccionar, para cada nivel, unos conocimientos mínimos que deben adquirir todos los alumnos para «demostrar que han aprendido», al margen del grupo concreto al que van dirigidos. 
Dentro de esta dimensión, llama la atención que no se hayan conseguido resultados estadísticamente significativos en la declaración que hace referencia a tomar las posibles ideas alternativas de los alumnos como fuente de conocimiento para secuenciar los contenidos (ítem 41 ), aspecto que se considera fundamental en la investigación actual en didáctica de las ciencias y que se menciona expresamente en la parte teórica del curso del CAP. También destaca el hecho de que los futuros profesores no reconocen de forma unánime la necesidad de utilizar una diversidad de fuentes a la hora de seleccionar los contenidos (ítem 43), aunque parece superada la idea de que la única fuente de información, a la hora, de seleccionar los contenidos, son los libros de texto (ítem 42).

\section{Dimensión «evaluación»}

Tal como se vio al describir el cuestionario, tres son los aspectos investigados relativos a esta dimensión (gráfico 2). En cuanto a los instrumentos a emplear para evaluar a los alumnos, destaca el consenso obtenido en las siguientes afirmaciones (ítems 1, 3 y 4): la evaluación de las materias científicas debe tener en cuenta los resultados obtenidos por los estudiantes en los trabajos prácticos (más del 90\%), hay que evaluar con más instrumentos que los tradicionales exámenes escritos $(75 \%)$ y se deben emplear pruebas específicas para valorar actitudes $(73 \%)$. En contra de las opinión de los profesores en ejercicio (Martínez et al., 1997), los futuros profesores no consideran que los alumnos estudien sólo para examinarse y encuentran posible hacer un seguimiento diario e individual de los estudiantes.

En el diseño y organización de la evaluación, se ha obtenido un pensamiento homogéneo en las siguientes cuestiones (ítems 9 y 11): un $90 \%$ de los futuros profesores manifiesta que permitir a los alumnos valorar los resultados de la evaluación va a ser una estrategia adecuada para conseguir un aprendizaje eficaz, afirmando además que «no se les debe exigir igual a todos los alumnos», ya que deben considerarse sus características individuales $(77 \%)$.

Por último, en la finalidad de la evaluación, hay que remarcar el acuerdo de un $72 \%$ de los estudiantes del CAP al reconocer que una parte importante de esta tarea debe referirse a contenidos de procedimiento (ítem 12). Esta manifestación es coherente con la ya comentada sobre la valoración académica que hay que efectuar de los trabajos prácticos realizados por el alumnado.

\section{Dimensión «metodología»}

En esta dimensión, en que por su extensión se han seleccionado cinco aspectos, son muchas las creencias que han obtenido consenso entre los futuros profesores y además con puntuaciones elevadas (Gráfico 3). Dentro de la planificación, el $88 \%$ destaca la necesidad de indagar las ideas previas con que los alumnos llegan a las aulas (ítem 13) y la idoneidad de organizar la asignatura en unidades didácticas diseñando actividades de inicia- ción, reestructuración de ideas y aplicación a nuevos contextos (ítems 14 y 15), todo ello enmarcado en una orientación claramente constructivista. No obstante, se puede detectar cierta contradicción entre estos resultados y el que comentamos anteriormente en la dimensión de contenido en que no había homogeneidad entre los estudiantes del CAP al considerar las ideas alternativas de los alumnos como fuente de conocimiento para el profesor, debido probablemente a una visión parcelada de lo que representa un currículo escolar y a su falta de experiencia.

Con respecto al desarrollo de la enseñanza, se manifiestan como mayoritarias afirmaciones relativas a la importancia de comprobar, con las actividades pertinentes, la reestructuración de esquemas alternativos $(80 \%$ en el ítem 17), la inclusión de implicaciones sociales en la enseñanza ( $87 \%$ en el ítem 18) y la conexión entre las actividades de aula y los problemas cotidianos que puedan preocupar a los estudiantes (96\% en el ítem 19).

Sobre la adaptación al alumno, los profesores en formación indican mayoritariamente la necesidad de diseñar actividades acordes con las capacidades de cada alumno, manifestando en un $73 \%$ la necesidad de poner especial atención en aquéllos que presentan problemas para aprender (ítem 23). Aseguran en un $80 \%$ que, planteando la enseñanza de esa forma, se conseguirán en los estudiantes actitudes favorables hacia el proceso del aprendizaje (ítem 65).

Con relación a la motivación y participación aparece una gran homogeneidad en un número elevado de ideas, poniendo de relieve la gran sensibilidad por parte de los profesores en formación hacia el alumno como persona (recordemos que ellos también son alumnos). Así, manifiestan unánimemente estar de acuerdo con revisar el método de enseñanza seguido en el aula cuando es criticado por los alumnos (91\%), motivar insistiendo en la utilidad de lo que se enseña $(98 \%)$ y programar la realización de pequeñas investigaciones por alumnos organizados en grupos (98\%). En otro orden de cosas, un $95 \%$ de los encuestados señala la necesidad de fomentar la colaboración entre compañeros y de inculcar en los alumnos la idea de que «es mejor cooperar que competir» para mejorar el aprendizaje $(92 \%)$. Estos resultados han sido también remarcados y valorados en el mismo sentido en otros estudios llevados a cabo con profesores en activo (Brincones et al., 1986; Rodrigo, 1994), lo que indica una cierta madurez en el pensamiento pedagógico de los futuros profesores en lo referente a aspectos de motivación y participación dentro de las aulas.

Mención aparte merece la afirmación de un significativo porcentaje de individuos, el $74 \%$, indicando que «los aspectos históricos pueden utilizarse como recurso motivador» (ítem 31), lo que aporta un dato más a un aspecto, el uso de la historia de la ciencia en la actividad docente, que se presenta como controvertido en distintos estudios: con alumnos de bachillerato (Carrascosa et al., 1991), con profesores de bachillerato (Brincones et al., 1986) y con profesores de enseñanza primaria (Rodrigo, 1994). 


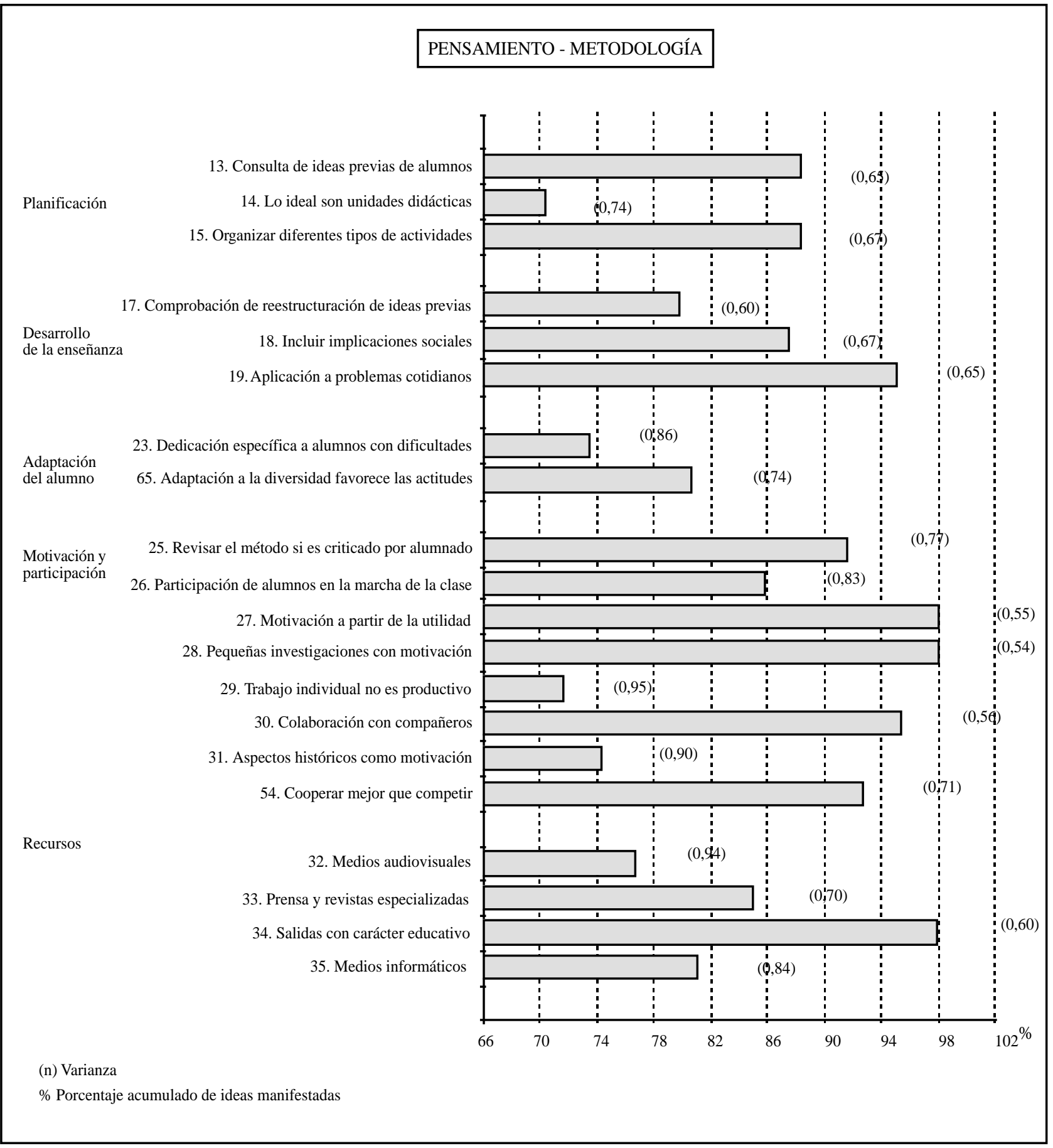

Para terminar, en el campo de los recursos, fundamental en la enseñanza de las ciencias experimentales, los estudiantes del CAP han mostrado su acuerdo sobre todas las proposiciones planteadas $(32,33,34$ y 35): utilidad de los medios audiovisuales e informáticos (alrededor de un 78\%), importancia del uso en las aulas 
de periódicos y de revistas especializadas (84\%) y necesidad de realizar salidas del centro escolar con carácter educativo $(98 \%)$.

Como resumen de los resultados podemos afirmar que los futuros profesores manifiestan una tendencia homogénea de pensamiento próxima a una visión constructivista del proceso de enseñanza-aprendizaje dentro del aula, pues, de las 28 cuestiones en que ha aparecido una uniformidad valorada como estadísticamente significativa, 27 están enmarcadas en el mencionado enfoque, lo que nos permite equiparar la muestra investigada con la de profesionales que han recibido una formación permanente cualificada (Rodrigo et al., 1999). Sin embargo, aparecen algunas manifestaciones de carácter más tradicional como, por ejemplo, en cuestiones relativas a la selección de contenidos, en las que han señalado la necesidad de programar un nivel de conocimientos mínimos para la generalidad de los alumnos al margen de su situación individual (79\% de acuerdo con la afirmación de la proposición 46). Además, si contrastamos la afirmación anterior con ideas aceptadas mayoritariamente sobre la evaluación (tal como la indicada en el ítem 11 en el cual el 90\% manifiesta su acuerdo en no exigir por igual a todos los alumnos), aparece cierta incoherencia en el pensamiento de los futuros profesores. Esta falta de coherencia interna en el pensamiento se puede detectar también cuando se compara la contestación a la cuestión 13 (un $88 \%$ ha considerado que es fundamental indagar las ideas alternativas de los alumnos) con las opiniones vertidas en los ítems 42 y 43 (una mayoría de los encuestados no considera que las citadas ideas tengan que ser una fuente de información a la hora de seleccionar los contenidos). Una explicación a estas contradicciones la podemos encontrar si tenemos en cuenta que estas personas tienen un conocimiento sobre la práctica docente elaborado a partir de su larga experiencia como alumnos, la cual «resiste» y entra en conflicto con los planteamientos teóricos que hayan podido asumir en su período de formación inicial (Mellado, 1998; De Pro, 1995; Furió et al., 1992; Valli, 1992). La superación de este conflicto es un aspecto que nosotros como formadores no deberíamos ignorar y que tiene que estar presente en cualquier diseño que se realice para formar futuros profesores.

En definitiva, los estudiantes futuros profesores han asumido gran parte de los planteamientos que han recibido en el proceso teórico de formación inicial en el cual han estado inmersos, situándose en posiciones próximas al enfoque constructivista, sobre todo en cuestiones claramente teóricas. Manifestaciones como, por ejemplo, «en la evaluación no hay que exigir por igual a todos los estudiantes de un determinado nivel» $\mathrm{O}$ «la necesidad por parte del profesorado de diseñar tareas y actividades específicas para los alumnos en función de sus características personales y sociales» son representativas de esta mentalidad. Esta conclusión estaría en línea con el llamado «optimismo pedagógico» que otros autores han puesto de manifiesto al estudiar el pensamiento pedagógico con muestras de futuros profesores de educación secundaria de distintas especialidades (Barquín, 1991; Pérez Gómez y Gimeno, 1992). No obstante, cuando se pasa del plano teórico a cuestiones relativas al ámbito del «saber hacer profesional», se ha detectado un acercamiento a las posturas defendidas por los profesores con enfoques más tradicionales sobre la enseñanza (Martínez et al., 1997; Rodrigo et al., 2000).

\section{ALGUNAS CONSIDERACIONES PARA LA FORMACIÓN INICIAL DEL PROFESORADO}

A tenor de los resultados de este estudio, y con todas las precauciones que de sus características se derivan, entendemos que, en la formación inicial del profesorado de ciencias de educación secundaria, sería necesario:

- Reflexionar sobre el contenido escolar que el futuro profesor tendrá que enseñar y sobre lo que sus alumnos saben y pueden aprender para poder realizar la transposición didáctica que permita elaborar conocimiento escolar a partir del conocimiento científico y cotidiano. La falta de reconocimiento, por parte de la muestra estudiada, de la diversidad de fuentes para la selección de los contenidos escolares nos induce a plantear la necesidad de un tratamiento didáctico de los contenidos de ciencias, en el sentido de analizar y transformar informaciones procedentes de fuentes diversas (la historia de la disciplina, los estudios sobre las ideas de los alumnos, los textos de divulgación, el conocimiento curricular ya elaborado, etc.) para convertirla en una propuesta de conocimiento escolar que se formule con diferentes niveles de complejidad (Martín del Pozo, 1998)

- Incrementar el tratamiento de las cuestiones epistemológicas relacionadas con el conocimiento científico y también con el conocimiento cotidiano. En este sentido, conviene profundizar en el estudio de las ideas de los alumnos de secundaria, no sólo desde el punto de vista de su utilización didáctica, que parece asumido por los futuros profesores, sino también de su naturaleza epistemológica y su relación con otras formas de conocimiento. Además, teniendo en cuenta los resultados obtenidos por nosotros con profesores en activo, éste es un aspecto de interés en la formación del profesorado que parece distinguir a los profesores con mayor nivel de desarrollo profesional (Rodrigo et al., 2000).

- Profundizar en las características de un metodología didáctica de corte constructivista e investigativo, que parece ser bien acogida por los futuros profesores, al menos en el plano declarativo, para que tenga una mayor incidencia en la práctica profesional tutorizada. Más concretamente, vemos la necesidad de diseñar y llevar a la práctica actividades de aprendizaje adaptadas a las características de cada alumno, la realización de pequeñas investigaciones y la utilización de la diversidad de recursos con los que puede contarse para la enseñanza de las ciencias. Todos ellos son aspectos que requieren de un importante aprendizaje profesional que puede ser realmente significativo y duradero para los futuros profesores.

- Diseñar instrumentos de evaluación ligados a situaciones reales de aula, que abarquen conocimientos concep- 
tuales, de procedimiento y de actitud y que permitan una individualización del proceso de enseñanzaaprendizaje, como demandan los futuros profesores. Esta necesidad también ha sido puesta en evidencia en otros estudios, tanto con profesores en activo (Brincones et al., 1986) como en formación (Pérez Gómez y Gimeno, 1992)

- Potenciar el diseño, la puesta en práctica y la evaluación de unidades didácticas. La aceptación por parte de los futuros profesores de la idea de organizar la asignatura en unidades didácticas es un buen punto de partida para integrar los diferentes elementos curriculares ya comentados. Como en el caso de los profesores en activo que han realizado cursos de actualización científica y didáctica (MEC, 1992), el diseño y desarrollo de unidades didácticas es una de las actividades formativas mejor valoradas y con mayores repercusiones en la actuación docente (De Pro, 1995; Martínez et al., 1997). En este sentido, como señalan muchos autores (Imbernón, 1994; Marcelo, 1991) es de vital importancia llevar a cabo esta actividad formativa por tutores expertos y con el estudio de casos reales.

- Reforzar adecuadamente las percepciones profesionales (responsabilidad ante la comunidad, necesidad de la formación psicopedagógica, confianza en las instituciones educativas, etc.) manifestadas por los futuros profesores. En caso contrario, al enfrentarse a la complejidad de la práctica pueden desarrollar estrategias de supervivencia en el aula, con hábitos negativos que son difíciles de erradicar, como algunos estudios parecen señalar (Marcelo, 1991, 1995; Mellado, 1998). Un mejor reconocimiento social de nuestra profesión también pasaría por potenciar, desde la formación inicial, la idea de que es necesario un continuo desarrollo profesional.

Desde nuestro punto de vista, estas necesidades formativas pueden sintetizarse en el gran reto de toda propuesta de formación: la relación teoría-práctica. Como señala Ferry (1983), debemos buscar planteamientos diferentes a la aplicación práctica de una teoría o a la teorización de la práctica. Para Gimeno (1988), la dialéctica entre la teorización y la práctica tiene una clara implicación en la formación inicial: incorporar la problemática del currículo escolar al contenido del currículo de los futuros profesores. Así, los problemas profesionales del currículo escolar pueden ser los organizadores de otros conocimientos disciplinares y los mediadores de aquéllos con la práctica. Las didácticas específicas pueden cumplir el papel de disciplinas prácticas capaces de cubrir ese espacio mediador e integrador entre la teoría y la práctica (Furió et al., 1992; Porlán, 1993; Blanco et al., 1995). No obstante, hemos de evitar:

a) un enfoque instrumentalista de la didáctica específica que podría provocar una desconexión entre el conoci- miento que proviene de las disciplinas de ciencias y el conocimiento didáctico sobre los contenidos de ciencias implicados en el currículo escolar;

b) el reduccionismo epistemológico que significa equiparar el conocimiento de las disciplinas de ciencias de la naturaleza con el conocimiento profesional para enseñar acerca de esas disciplinas o con el conocimiento generado por la didáctica de las ciencias como disciplina emergente (Bromme, 1988; Porlán, 1998);

c) la yuxtaposición de dos discursos que no se complementan: un discurso teórico atribuido al profesor de la universidad y un discurso práctico atribuido a los profesores de secundaria.

Afortunadamente, la integración entre la formación teórica y la formación práctica es también el planteamiento formativo esencial del nuevo curso de cualificación pedagógica. Todo ello se intenta conseguir a través de un currículo con materias de carácter psicopedagógico y didáctico y práctica profesional docente tutorizada, con lo que se pretende conseguir un profesorado «altamente cualificado» con una formación inicial «amplia, sólida y rigurosa», para después proseguir su desarrollo profesional con una formación permanente adecuada (Real Decreto 1692/1995 de 20 de Octubre)

En definitiva, lo que se propone es facilitar la construcción de un conocimiento profesional significativo y riguroso, cercano a la realidad escolar, para consolidar los planteamientos más teóricos que los futuros profesores parecen asumir con facilidad. Esto supone incorporar los problemas relativos al diseño y al desarrollo del currículo escolar como contenido de la formación inicial, en un proceso que permita avanzar desde el nivel en que los profesores se encuentren hasta llegar a ser un profesional autónomo, reflexivo, crítico e investigador. Dicho en otros términos, el desarrollo profesional que empieza con la formación inicial debe tender gradualmente a que el profesor sea un facilitador del aprendizaje significativo de los alumnos y un investigador de los procesos de enseñanza-aprendizaje que se dan en su aula.

\section{NOTAS}

* Esta publicación es parte del trabajo titulado Estudio de la incidencia de distintos programas de formación y de la práctica educativa en el pensamiento del profesor de ciencias de educación secundaria, financiado con cargo a la convocatoria 1995 de ayudas a la investigación educativa del CIDE.

* Agradecemos a la dirección y a los profesores del CAP las facilidades proporcionadas para la aplicación del cuestionario, y a los estudiantes, su colaboración desinteresada. 


\section{REFERENCIAS BIBLIOGRÁFICAS}

AZORÍN, F. y SÁNCHEZ CRESPO, J. L. (1986). Métodos y aplicaciones del muestreo. Madrid: Alianza.

BARQUÍN, J. (1991). La evolución del pensamiento del profesor. Revista de Educación, 294, pp. 245-274.

BARQUÍN, J. (1995). La investigación sobre el profesorado. Estado de la cuestión en España. Revista de Educación, 306, pp. 7-65.

BERNSTEIN, B. (1988). Clases, códigos y control. Madrid: Akal.

BLANCO,L.J., MELLADO, V.y RUIZ, C. (1995). Conocimiento didáctico del contenido en ciencias experimentales y matemáticas y formación de profesores. Revista de Educación, 307, pp. 427-446.

BRINCONES, I., FUENTES, A., NIEDA, J., PALACIOS, M J. y OTERO, J. (1986). Identificación de comportamientos deseables del profesorado de ciencias experimentales del bachillerato. Enseñanza de las Ciencias, 4(3), pp. 209-222.

BROADFOOT, P. y OSBORN, M. (1988). What professional responsability means to teachers: national contexts and classrooms constants. British Journal of Sociology of Education, 9(3), pp. 265-287.

BROMME, R. (1988). Conocimientos profesionales de los profesores. Enseñanza de las Ciencias, 6(1), pp. 19-29.

CARRASCOSA, J., FERNÁNDEZ, I., GIL, D. y OROZCO, A. (1991). La visión de los alumnos sobre lo que el profesorado de ciencias ha de saber y saber hacer. Investigación en la escuela, 14, pp. 45-61.

COLL, C. (1994). De qué hablamos cuando hablamos de constructivismo. Cuadernos de Pedagogía, 221, pp. 8-10.

CRONIN-JONES, L. (1991). Science teacher beliefs and their influence on curriculum implementation: two case studies. Journal of Research in Science Teaching, 28(3), pp. 235-250.

DE PRO, A. (1995).Formación de profesor de secundaria versus profesor tutor de prácticas de Secundaria, en Blanco, L.J. y Mellado, V. (coords.). La formación del profesorado de ciencias y matemáticas en España y Portugal. Badajoz: Universidad de Extremadura, pp. 375-397.

DESAULTES, S. (1993). La formation à l'enseignement des sciences: le virage épistemologique. Didaskalia, 1, pp. 49-67.

DRIVER, R., GUESNE, E. y TIBERGHEIN, A. (1989). Ideas científicas en la infancia y la adolescencia. Madrid: MorataMEC.

ESTEVE, J.M. (1997). La formación inicial de los profesores de secundaria. Barcelona: Ariel.

FERNÁNDEZ, J. y ELORTEGUI, N. (1996). Qué piensan los profesores acerca de cómo se debe enseñar. Enseñanza de las Ciencias, 14(3), pp. 331-342.

FERRY, G. (1983). Le trajet de la formation. París: Dunod.

FURIÓ, C. (1994). Tendencias actuales en la formación del profesorado de ciencias. Enseñanza de las Ciencias, 12(2), pp. 188-199.

FURIÓ, C., GIL, D., PESSOA, A.M. y SALCEDO, L.E. (1992). La formación inicial del profesorado de educación secundaria. Investigación en la escuela, 16, pp. 7-21.
GALLAGHER, J.J. (1993). Six Views of Teaching Science. An Invitation to Reflection and Discussion. Michigan State University. (Documento multicopiado)

GALLAGHER, J.J.(1991). Prospective and practicing secondary school science teachers' knowledge and belief about the philosophy of science. Science Education ,75(1), pp. 121133.

GARCÍA YAGÜE, E. (1988). Condición social y feminización del profesorado de educación básica. Revista de Educación, 285 , pp. $244-267$.

GIL, D. (1991). ¿Qué hemos de saber y saber hacer los profesores de ciencias? (Intento de síntesis de las aportaciones de la investigación didáctica). Enseñanza de las Ciencias, 9(1), pp. 69-77.

GIMENO, J. (1988). El curriculum: una reflexión sobre la práctica. Madrid: Morata.

GUERRERO SERÓN, A. (1997). El perfil socioprofesional del profesorado de enseñanza media y su actitud ante la reforma de la enseñanza secundaria. Revista de Educación, 314, pp. 247-266.

GUÍA DE ESTUDIO PARA EL CAP. (1997). Madrid: ICE de la UCM.

GUNSTONE, R.F., SLATTERY, M., BAIR, J.R. y NORTHFIELD, J.R. (1993). A case study exploration development in preservice science teachers. Science Education, 77(1), pp. 47-73.

GUSTAFSON, B.J. y ROWELL, P.M. (1995). Elementary preservice teachers: constructing conceptions about learning science, teaching science and the nature of science. International Journal of Science Education, 17(5), pp. 589-605.

HASHWEH, M.Z. (1996). Effects of Science Teachers' Epistemological Beliefs in Teaching. Journal of Research in Science Teaching, 33(1), pp. 47-63.

HEWSON, P., KERBY, H. y COOK, P. (1995).Determining the conceptions of teaching science held by experienced high school science teachers. Journal of Research in Science Teaching, 32(5), pp. 503-520.

HEWSON, P.W. y HOLLON, R. (1994).Connecting thought and action in high school science classrooms. WisconsinMadison: Wisconsin Center for Education Research.

HOLLON, R.E., ROTH, K.J. y ANDERSON, C.W. (1991). Science teachers' conceptions of teaching and learning. Advances in Research on Teaching, 2, pp. 145-185.

HOLLON, R.E. y ANDERSON, C.W.(1987). Teachers' beliefs abouts students'learning processes in science: self-reinforcing belief systems. Documento presentado en Annual Meeting of the AERA. Washington, DC.

IMBERNÓN, F. (1994). La formación y el desarrollo profesional del profesor. Barcelona: Graó.

KENNEDY, M.M. (1991). An agenda for research on teacher learning. Michigan Estate University: Nacional Center for Research on Teacher Learning.

KNOWLES, G. (1992). Models for understanding preservice and beginning teachers' biographies: Illustration from case studies, en Goodson, I. (ed.) Studying Teacher Lives, pp. 99-152. Londres: Routledge.

KOULADIS, V. y OGBORN, J. (1995). Science teachers' philosophical assumptions: how well do we understand 
them? International Journal of Science Education, 17(3), pp. 273-283.

KOULADIS, V. y OGBORN, J. (1989). Philosophy of science: an empirical study of teachers' views. International Journal of Science Education, 11(2), pp. 173-184.

LEDERMAN, N.G. (1992). Student's and teachers'conceptions of the nature of science: a review of the research. Journal of Research in Science Teaching, 29(4), pp. 331-359.

LEDERMAN, N.G. y O'MALLY, M. (1990). Students' percepctions of tentativeness in science: Development, use and sources of change. Science Education, 74(2), pp. 225239.

LÓPEZ, J.I.(1995). «El conocimiento profesional de los profesores acerca de las concepciones de los alumnos. Dos estudios de caso en la enseñanza y aprendizaje de las ciencias». Tesis doctoral. Universidad de Sevilla.

MARCELO, C. (1995). Investigaciones sobre formación del profesorado: el conocimiento sobre el aprender a enseñar, en Blanco, L.J. y Mellado, V. (coords.). La formación del profesorado de ciencias y matemáticas en España y Portugal. Badajoz: Universidad de Extremadura.

MARCELO, C. (1991). Aprender a enseñar. Un estudio sobre el proceso de socialización de profesores principiantes. Madrid: CIDE.

MARRERO, J. (1993). Las teorías implícitas del profesorado: vínculo entre la cultura y la práctica de enseñanza, en Rodrigo, M.J., Rodríguez, A. y Marrero, J. (eds.). Las teorías implícitas. Una aproximación al conocimiento cotidiano. Madrid: Visor.

MARTÍN DEL POZO, R.(1998). La construcción didáctica del concepto de cambio químico. Alambique, 17, pp. 65-75.

MARTÍN DEL POZO, R. (1994). «El conocimiento del cambio químico en la formación inicial del profesorado. Estudio de las concepciones disciplinares y didácticas de los estudiantes de magisterio». Tesis doctoral inédita. Universidad de Sevilla.

MARTÍNEZ, M. M., FERNÁNDEZ, M. P., GUERRERO, A., MARTÍN, R., RODRIGO, M. y VARELA, M.P. (1997). «Estudio de la incidencia de distintos programas de formación y de la práctica educativa en el pensamiento del profesor de ciencias de educación secundaria». Memoria de investigación inédita. Madrid: CIDE.

MEC (1992). Curso de actualización científica y didáctica. Ciencias de la naturaleza. (Carpeta de varias monografías). Madrid: MEC.

MELLADO, V. (1996). Concepciones y prácticas de aula de profesores de ciencias en formación inicial de primaria y secundaria. Enseñanza de las Ciencias, 14(3), pp. 298-302.

MELLADO, V. (1998).El estudio de aula en la formación continua del profesorado de ciencias. Alambique, 15, pp. 39-46.

ORLANDI, E. (1991). Conceptions des enseignants sur la démarche expérimentale. Analyse de quelques cas à propos de digestion en classe de troisième. Aster, 13, pp. 11-132.

PÉREZ GÓMEZ, A. I. y GIMENO, J. (1992). El pensamiento pedagógico de los profesores: un estudio empírico sobre la incidencia de los cursos de aptitud pedagógica (CAP) y de la experiencia profesional en el pensamiento de los profesores. Investigación en la Escuela, 17, pp. 51-73.

POMEROY, D. (1993). Implications of teachers' beliefs about the nature of science: comparison of the beliefs of scientists, secondary science teachers, an elementary teachers. Science Education, 77(3), pp. 261-278.
POPE, M. y SCOTT, E.M. (1988). La epistemología y la práctica de los profesores, en Porlán, R., García, J.E. y Canal, P. (eds.). Constructivismo y enseñanza de las ciencias. Sevilla: Díada.

PORLÁN, R. (1998). Pasado, presente y futuro de la didáctica de las ciencias. Enseñanza de las Ciencias, 16(1), pp. 175185.

PORLÁN, R. (1993). Constructivismo y escuela. Sevilla: Díada.

PORLÁN, R. (1989). «Teoría del conocimiento, teoría de la enseñanza y desarrollo profesional. Las concepciones epistemológicas de los profesores». Tesis doctoral. Universidad de Sevilla.

PORLÁN, R. y RIVERO, A. (1998). El conocimiento profesional de los profesores. Sevilla: Díada.

PORLÁN, R., RIVERO, A. y MARTÍN DEL POZO, R. (1998). Conocimiento profesional y epistemología de los profesores II: Estudios empíricos y conclusiones. Enseñanza de las Ciencias, 16(2), pp. 271-288.

PORLÁN, R., RIVERO, A. y MARTÍN DEL POZO, R. (1997). Conocimiento profesional y epistemología de los profesores I: Teoría, métodos e instrumentos. Enseñanza de las Ciencias, 15(2), pp. 155-171.

PORLÁN, R. y MARTÍN DEL POZO, R. (1996). Ciencia, profesores y enseñanza: unas relaciones complejas. Alambique, 8, pp. 23-32.

PRAWAT, R.S. (1992).Teachers' beliefs about teaching and learning: a constructivist perspective. American Journal of Education, 100(3), pp. 354-395.

RODRIGO, M., MARTÍN, R., MARTÍNEZ, M.M., VARELA, M.P., FERNÁNDEZ, M.P. y GUERRERO, A. (2000). Un estudio sobre el profesor de ciencias de educación secundaria y unas propuestas para mejorar su formación. Revista de Educación, 321. (En prensa.)

RODRIGO, M.(1994). Aproximaciónal pensamientodel profesor de ciencias de la ESO. Revista Complutense de Educación, 5(2), pp. 271-288.

SHULMAN, L.S. (1986). Paradigms and Research Programs in the Study of Teaching: A Contemporary Perspective, en Wittrock, M.C. (ed.). Handbook of Research on Teaching. Nueva York: Macmillan. Trad. cast. (1989). Paradigmas y programas de investigación en el estudio de la enseñanza: una perspectiva contemporánea, en La investigación de la enseñanza (I). Enfoques y teorías. Barcelona: MEC-Paidós.

SMITH, D. y NEALE, D. (1991). The Construction of SubjetMatter Knowledge in Primary Scienc Teaching, en Brophy, J. (ed.). Advances in Research on Teaching, 2. JAI Press.

TRIANDIS, H.C. (1971). Attitudes and Attitudes Change. Nueva York: John Wiley.

WIDEEN, M., MAYER-SMITH, J. y MOON, B. (1998). A Critical Analysis of the Research on Learning to Teach: Making the Case for an Ecological Perspective on Inquiry. Review of Educational Research, 68(2), pp. 130-178.

YINGER, R.J. (1986). Investigación sobre el conocimiento y pensamiento de los profesores. Hacia una concepción de la actividad profesional, en Actas I Congreso Internacional sobre Pensamientos de los Profesores y Toma de Decisiones. Sevilla: Servicio de publicaciones de la Universidad de Sevilla.

VALLI, L. (1992). Begining teacher problems: Areas for Teacher Education Improvement. Action in Teacher Education, 14(1), pp. $18-25$.

[Artículo recibido en abril de 1999 y aceptado en diciembre de 1999.] 


\section{ANEXO}

\section{CUESTIONARIO}

\section{I . DATOS DE IDENTIFICACIÓN}

a) EDAD: Señale el grupo de edad en que se encuentra:
1) 25 años o menos
5) 41-45 años
2) $26-30$ años
6) 46-50 años
3) 31-35 años
7) 51-55 años
4) 36-40 años
8) 56 o más años

b) SEXO

1) Mujer

2) Hombre

c) ¿Podría indicar cuál es o ha sido la ocupación principal (en activo) de su padre y de su madre?

-Ocupación principal del padre:

-Ocupación principal de la madre:

d) Indique, por favor, qué estudios tiene Ud. terminados?

-Magisterio o profesor de EGB

-Diplomatura en:

-Licenciatura en:

Física

Química

Biología

Geología

Ingeniería o arquitectura:

-Otras:

- Doctorado en:

e) Le pedimos ahora que señale el grado de influencia que ejercen sobre el trabajo docente cada uno de los factores que aparecen a continuación

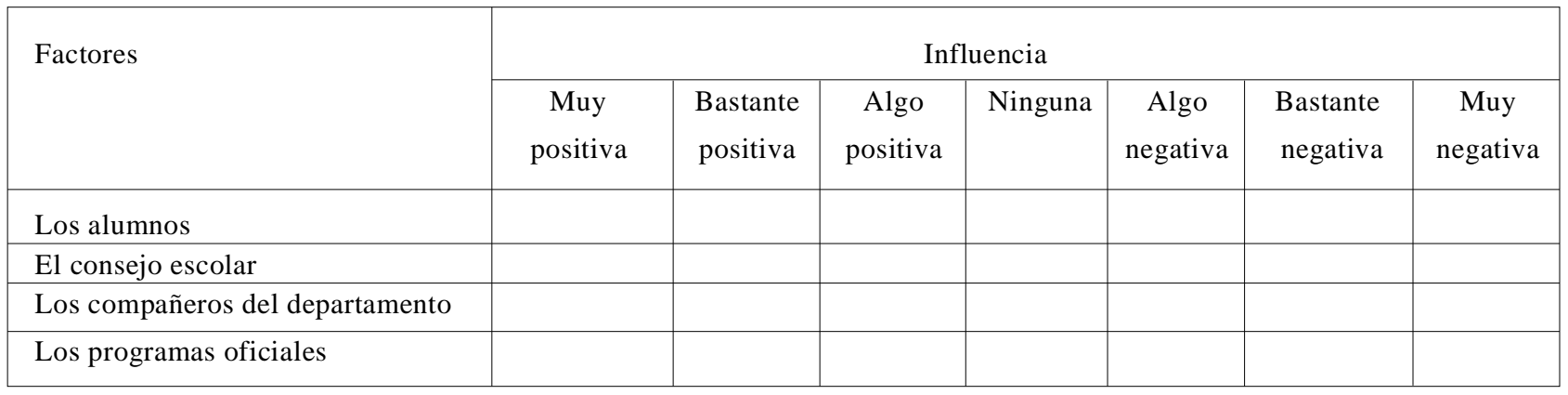


INVESTIGACIÓN DIDÁCTICA

\begin{tabular}{|l|l|l|l|l|l|l|}
\hline La inspección & & & & & & \\
\hline Los libros de texto & & & & & & \\
\hline El director del centro & & & & & & \\
\hline Los padres y las madres de alumnos & & & & & & \\
\hline $\begin{array}{l}\text { La responsabilidad profesional de los } \\
\text { profesores }\end{array}$ & & & & & & \\
\hline El claustro de profesores & & & & & & \\
\hline $\begin{array}{l}\text { Los cursos de actualización } \\
\text { y reciclaje }\end{array}$ & & & & & & \\
\hline
\end{tabular}

f) En realidad, ¿cómo cree Ud. que la actitud de los siguientes grupos o realidades sociales inciden sobre el nivel del alumnado?

\begin{tabular}{|l|l|l|l|}
\hline & LO SUBEN & LO MANTIENEN & LO BAJAN \\
\hline La programación de TV & & & \\
\hline El D.C.B. de ciencias & & & \\
\hline La colaboración de los padres & & & \\
\hline La generalización de la secundaria & & & \\
\hline El profesorado de primaria & & & \\
\hline El profesorado de secundaria & & & \\
\hline La falta de perspectivas de empleo & & & \\
\hline
\end{tabular}




\section{CUESTIONARIO SOBRE EL PE NSAMIENTO EDUCATIVO *}

\begin{tabular}{|c|c|c|c|c|c|}
\hline & $\begin{array}{l}\text { Totalmente } \\
\text { de acuerdo }\end{array}$ & $\begin{array}{c}\text { De } \\
\text { acuerdo }\end{array}$ & Indeciso & $\begin{array}{c}\text { En } \\
\text { desacuerdo }\end{array}$ & $\begin{array}{l}\text { Totalmente } \\
\text { en desacuerdo }\end{array}$ \\
\hline 1. La clave de una correcta evaluación es el examen escrito. & & & & & \\
\hline 2. Si no hubiera exámenes, los alumnos no estudiarían. & & & & & \\
\hline $\begin{array}{l}\text { 3. Es imprescindible resaltar la evaluación de las actitudes en } \\
\text { la nota final. }\end{array}$ & & & & & \\
\hline $\begin{array}{l}\text { 4. La calificación del trabajo práctico debe constituir una parte } \\
\text { importante de la nota de cada evaluación. }\end{array}$ & & & & & \\
\hline $\begin{array}{l}\text { 5. En nuestras aulas no es posible hacer un seguimiento diario } \\
\text { e individual de cada alumno. }\end{array}$ & & & & & \\
\hline $\begin{array}{l}\text { 6. Las pruebas de evaluación deben ser preparadas } \\
\text { individualmente por cada profesor, pues es quien conoce su } \\
\text { clase. }\end{array}$ & & & & & \\
\hline $\begin{array}{l}\text { 7. El diseño de las pruebas de evaluación debe hacerse siempre } \\
\text { de forma colegiada. }\end{array}$ & & & & & \\
\hline $\begin{array}{l}\text { 8. El objetivo principal de la evaluación es comprobar si se ha } \\
\text { alcanzado el nivel de conocimientos previsto para la clase. }\end{array}$ & & & & & \\
\hline $\begin{array}{l}\text { 9. Para un aprendizaje eficaz, resulta adecuado permitir a los } \\
\text { alumnos que valoren los resultados de la evaluación. }\end{array}$ & & & & & \\
\hline $\begin{array}{l}\text { 10. Uno de los objetivos más importantes de la evaluación es } \\
\text { conseguir que cada alumno sea consciente de sus dificultades. }\end{array}$ & & & & & \\
\hline $\begin{array}{l}\text { 11. A todos los alumnos se les debe exigir igual sin atender a } \\
\text { sus características individuales. }\end{array}$ & & & & & \\
\hline $\begin{array}{l}\text { 12. Una parte importante de cada evaluación deberá considerar } \\
\text { objetivos referidos a los procedimientos. }\end{array}$ & & & & & \\
\hline $\begin{array}{l}\text { 13. Antes de iniciar un bloque, debería indagarse las ideas de } \\
\text { los alumnos para organizar el aprendizaje en consecuencia. }\end{array}$ & & & & & \\
\hline $\begin{array}{l}\text { 14. En la planificación de la enseñanza, lo más adecuado es } \\
\text { utilizar unidades didácticas elaboradas por grupos de profesores. }\end{array}$ & & & & & \\
\hline $\begin{array}{l}\text { 15. La planificación del trabajo de aula debe incluir que los } \\
\text { alumnos realicen actividades de iniciación, de desarrollo, de } \\
\text { reestructuración y de aplicación. }\end{array}$ & & & & & \\
\hline $\begin{array}{l}\text { 16. Cada tema debería explicarse siguiendo un libro de texto } \\
\text { o apuntes claros. }\end{array}$ & & & & & \\
\hline $\begin{array}{l}\text { 17. Un número significativo de las actividades que se hacen } \\
\text { en el aula debería comprobar si las ideas iniciales de los } \\
\text { alumnos cambian. }\end{array}$ & & & & & \\
\hline $\begin{array}{l}\text { 18. Las implicaciones sociales de las ciencias deberían incluirse } \\
\text { en las actividades que se realizan en clase. }\end{array}$ & & & & & \\
\hline $\begin{array}{l}\text { 19. Una parte importante de las actividades que se realizan } \\
\text { debería buscar la aplicación de lo aprendido a problemas } \\
\text { cotidianos. }\end{array}$ & & & & & \\
\hline $\begin{array}{l}\text { 20. Las actividades prácticas deben servir, fundamentalmente, } \\
\text { para comprobar } a \text { posteriori los aspectos explicados } \\
\text { teóricamente. }\end{array}$ & & & & & \\
\hline $\begin{array}{l}\text { 21. Conviene que todos los alumnos dispongan del mismo } \\
\text { tiempo para realizar las actividades de aprendizaje. }\end{array}$ & & & & & \\
\hline
\end{tabular}




\begin{tabular}{|c|c|c|c|c|c|}
\hline & $\begin{array}{l}\text { Totalmente } \\
\text { de acuerdo }\end{array}$ & $\begin{array}{c}\text { De } \\
\text { acuerdo }\end{array}$ & Indeciso & $\begin{array}{c}\text { En } \\
\text { desacuerdo }\end{array}$ & $\begin{array}{l}\text { Totalmente } \\
\text { en desacuerdo }\end{array}$ \\
\hline $\begin{array}{l}\text { 22. Resulta utópico utilizar la estrategia metodológica que se } \\
\text { adapte mejor a cada grupo de alumnos en cada situación. }\end{array}$ & & & & & \\
\hline $\begin{array}{l}\text { 23. Es razonable diseñar tareas y actividades específicas para } \\
\text { los alumnos en función de sus características personales y } \\
\text { sociales. }\end{array}$ & & & & & \\
\hline $\begin{array}{l}\text { 24. Se debe permitir a los alumnos tomar algunas decisiones } \\
\text { sobre qué estudiar. }\end{array}$ & & & & & \\
\hline $\begin{array}{l}\text { 25. El profesor debería revisar su método de enseñanza si } \\
\text { éste fuera cuestionado por el alumnado. }\end{array}$ & & & & & \\
\hline $\begin{array}{l}\text { 26. Los alumnos deberían participar en el desarrollo de la } \\
\text { enseñanza en el aula. }\end{array}$ & & & & & \\
\hline $\begin{array}{l}\text { 27. Una forma de conseguir la motivación de los alumnos } \\
\text { es que vean la «utilidad práctica» de lo que aprenden. }\end{array}$ & & & & & \\
\hline $\begin{array}{l}\text { 28. Una manera de motivar es propiciar que los alumnos } \\
\text { realicen pequeñas investigaciones en el aula. }\end{array}$ & & & & & \\
\hline 29. El trabajo más productivo, para los alumnos, es el individual. & & & & & \\
\hline $\begin{array}{l}\text { 30. Es indispensable poner a disposición de los colegas los } \\
\text { materiales y la propia experiencia para mejorar la práctica } \\
\text { educativa. }\end{array}$ & & & & & \\
\hline $\begin{array}{l}\text { 31. Durante el curso se deberían utilizar, frecuentemente, } \\
\text { aspectos históricos de la ciencia sólo como un recurso } \\
\text { motivado. }\end{array}$ & & & & & \\
\hline $\begin{array}{l}\text { 32. Las nuevas tecnologías son imprescindibles en la enseñanza } \\
\text { de las ciencias, especialmente los medios audiovisuales. }\end{array}$ & & & & & \\
\hline $\begin{array}{l}\text { 33. Fuentes de información como la prensa escrita y revistas } \\
\text { especializadas son recursos fundamentales en las clases de } \\
\text { ciencias. }\end{array}$ & & & & & \\
\hline $\begin{array}{l}\text { 34. A lo largo del curso se deberían realizar salidas con carácter } \\
\text { educativo. }\end{array}$ & & & & & \\
\hline $\begin{array}{l}\text { 35. Las nuevas tecnologías son imprescindibles en la enseñanza } \\
\text { de las ciencias, especialmente los medios informáticos. }\end{array}$ & & & & & \\
\hline $\begin{array}{l}\text { 36. Los contenidos escolares no deben diferenciarse del } \\
\text { conocimiento científico. }\end{array}$ & & & & & \\
\hline $\begin{array}{l}\text { 37. Los contenidos escolares son una forma peculiar de } \\
\text { conocimiento, distinta al conocimiento científico y al } \\
\text { conocimiento ordinario. }\end{array}$ & & & & & \\
\hline $\begin{array}{l}\text { 38. El conocimiento científico es la forma de conocimiento } \\
\text { objetiva y correcta. }\end{array}$ & & & & & \\
\hline $\begin{array}{l}\text { 39. El conocimiento científico es producto de la actividad } \\
\text { humana, del contexto y de la cultura en que se desarrolla } \\
\text { y se usa. }\end{array}$ & & & & & \\
\hline $\begin{array}{l}\text { 40. Las ideas de los alumnos sobre los conceptos de ciencias } \\
\text { son errores que el profesor debe eliminar. }\end{array}$ & & & & & \\
\hline $\begin{array}{l}\text { 41. Las ideas de los alumnos sobre los conceptos de ciencias } \\
\text { son un conocimiento alternativo con el que hay que trabajar } \\
\text { en clase. }\end{array}$ & & & & & \\
\hline
\end{tabular}




\begin{tabular}{|c|c|c|c|c|c|}
\hline & $\begin{array}{l}\text { Totalmente } \\
\text { de acuerdo }\end{array}$ & $\begin{array}{c}\text { De } \\
\text { acuerdo }\end{array}$ & Indeciso & $\begin{array}{c}\text { En } \\
\text { desacuerdo }\end{array}$ & $\begin{array}{l}\text { Totalmente } \\
\text { en desacuerdo }\end{array}$ \\
\hline \multicolumn{6}{|l|}{$\begin{array}{l}\text { 42. Los libros de texto son la fuente de información funda- } \\
\text { mental para seleccionar los contenidos que hay que enseñar. }\end{array}$} \\
\hline \multicolumn{6}{|l|}{$\begin{array}{l}\text { 43. Para seleccionar y secuenciar los contenidos escolares } \\
\text { hay que tener en cuenta la información de los estudios } \\
\text { de las ideas de los alumnos, de la historia de la ciencia } \\
\text { y de otros materiales curriculares. }\end{array}$} \\
\hline \multicolumn{6}{|l|}{$\begin{array}{l}\text { 44. La forma más adecuada de organizar los contenidos } \\
\text { escolares sería la de seguir una secuencia lineal de temas. }\end{array}$} \\
\hline \multicolumn{6}{|l|}{$\begin{array}{l}\text { 45. Los mapas conceptuales son una de las formas más } \\
\text { adecuadas de organizar los contenidos escolares. }\end{array}$} \\
\hline \multicolumn{6}{|l|}{$\begin{array}{l}\text { 46. Hay un nivel de conocimientos genéricos al que deben } \\
\text { llegar los alumnos para demostrar que han «aprendido». }\end{array}$} \\
\hline \multicolumn{6}{|l|}{$\begin{array}{l}\text { 47. Los alumnos deben ser evaluados positivamente si hay } \\
\text { una evolución favorable de sus propias ideas, aunque no } \\
\text { alcancen el nivel deseable. }\end{array}$} \\
\hline \multicolumn{6}{|l|}{$\begin{array}{l}\text { 48. Actualmente, la formación científica recibida en la } \\
\text { universidad es suficiente para poder desempeñar la labor } \\
\text { docente en la enseñanza secundaria. }\end{array}$} \\
\hline \multicolumn{6}{|l|}{$\begin{array}{l}\text { 49. El profesorado de enseñanza secundaria necesita una } \\
\text { formación psicopedagógica complementaria a su formación } \\
\text { científica para desempeñar su función. }\end{array}$} \\
\hline \multicolumn{6}{|l|}{$\begin{array}{l}\text { 50. La procedencia socioeconómica de los alumnos es la principal } \\
\text { razón de su rendimiento escolar. }\end{array}$} \\
\hline \multicolumn{6}{|l|}{$\begin{array}{l}\text { 51. Aunque chicas y chicos parecen tener la misma } \\
\text { capacidad para el aprendizaje de las ciencias, las chicas } \\
\text { muestran una actitud más favorable hacia las mismas. }\end{array}$} \\
\hline \multicolumn{6}{|l|}{$\begin{array}{l}\text { 52. La mayor inclinación que presentan los chicos respecto } \\
\text { a las chicas en las disciplinas de ciencias refleja sus } \\
\text { mejores aptitudes en esta área. }\end{array}$} \\
\hline \multicolumn{6}{|l|}{$\begin{array}{l}\text { 53. En el área de ciencias es más importante poseer una } \\
\text { buena aptitud que en otras áreas curriculares. }\end{array}$} \\
\hline \multicolumn{6}{|l|}{$\begin{array}{l}\text { 54. Los alumnos aprenden mejor cuando cooperan que } \\
\text { cuando compiten. }\end{array}$} \\
\hline \multicolumn{6}{|l|}{$\begin{array}{l}\text { 55. El éxito o fracaso de los alumnos depende básicamente } \\
\text { de sus características personales (inteligencia, motivación...). }\end{array}$} \\
\hline \multicolumn{6}{|l|}{$\begin{array}{l}\text { 56. Un profesor de ciencias dispone de recursos suficientes } \\
\text { para hacer rendir adecuadamente a los alumnos con } \\
\text { independencia de la extracción social o características } \\
\text { personales de éstos. }\end{array}$} \\
\hline \multicolumn{6}{|l|}{$\begin{array}{l}\text { 57. El mayor fracaso de los alumnos en las asignaturas } \\
\text { de ciencias se debe fundamentalmente a que en su enseñanza } \\
\text { se utilizan métodos inadecuados. }\end{array}$} \\
\hline \multicolumn{6}{|l|}{$\begin{array}{l}\text { 58. La adaptación de la enseñanza a la diversidad del aula } \\
\text { reduce el nivel de los conocimientos en las materias } \\
\text { de ciencias. }\end{array}$} \\
\hline $\begin{array}{l}\text { 59. Tener en cuenta la diversidad de los alumnos a la hora } \\
\text { de impartir las materias de ciencias perjudica a los alumnos } \\
\text { más capacitados. }\end{array}$ & & & & & \\
\hline
\end{tabular}




\begin{tabular}{|c|c|c|c|c|c|}
\hline & $\begin{array}{l}\text { Totalmente } \\
\text { de acuerdo }\end{array}$ & $\begin{array}{c}\text { De } \\
\text { acuerdo }\end{array}$ & Indeciso & $\begin{array}{c}\text { En } \\
\text { desacuerdo }\end{array}$ & $\begin{array}{l}\text { Totalmente } \\
\text { en desacuerdo }\end{array}$ \\
\hline $\begin{array}{l}\text { 60. La personalidad y las actitudes del profesor de ciencias } \\
\text { tiene menor incidencia en el rendimiento de los alumnos que } \\
\text { sus conocimientos científicos y didácticos. }\end{array}$ & & & & & \\
\hline $\begin{array}{l}\text { 61. Las dificultades de las materias de ciencias se deben } \\
\text { en gran medida al lenguaje técnico propio de estas } \\
\text { disciplinas. }\end{array}$ & & & & & \\
\hline $\begin{array}{l}\text { 62. El desarrollo cognitivo de los alumnos es el principal } \\
\text { responsable de su rendimiento escolar. }\end{array}$ & & & & & \\
\hline $\begin{array}{l}\text { 63. Una de las finalidades importantes de las asignaturas de } \\
\text { ciencias es potenciar el pensamiento crítico de los alumnos. }\end{array}$ & & & & & \\
\hline $\begin{array}{l}\text { 64. Del conjunto de las áreas curriculares, las asignaturas de } \\
\text { ciencias son las que contribuyen, en mayor medida, } \\
\text { al ejercicio de la mente. }\end{array}$ & & & & & \\
\hline $\begin{array}{l}\text { 65. La adaptación de la enseñanza a la diversidad del aula } \\
\text { contribuye a generar actitudes más favorables hacia las } \\
\text { ciencias. }\end{array}$ & & & & & \\
\hline
\end{tabular}

\footnotetext{
* Notas

- Las opciones seleccionadas y sombreadas corresponden a la denominada tendencia constructivista.

- Las preguntas que aquí aparecen ordenadas, para su mejor consulta, en las encuestas estaban distribuidas al azar.
} 